\title{
Effects of Titanium in Alnico 8-Type Magnet Alloys*
}

\author{
By Yoshiro Iwama**, Matsumi Inagaki** \\ and Toshinobu Miyamoto****
}

\begin{abstract}
Alnico 8-type magnet alloys containing more than $5 \% \mathrm{Ti}$ have been well-known to have very high coercive forces, particularly when the isothermal field treatment is applied. As a primary approach in this research to explore its cause, the transformations, microstructures and magnet characteristics were investigated in a series of Alnico 8-type alloys with various titanium contents. The critical temperatures of the transformations including magnetic transition were determined as a function of titanium content. It may be reasonably concluded that the mechanism of magnetic hardening in these alloys is similar to that in Alnico 5-type alloys containing no titanium.

It was shown that when the alloys were subjected to isothermal field treatment, the coercive fore obtained depended largely upon the temperature of the treatment, and slightly upon the treating time. In each of the specimens, the optimum temperature of the treatment was found to exist about $50^{\circ} \mathrm{C}$ below the critical temperature of the two-phase separation reaction and to raise as the titanium content was increased. In addition, the magnetic anisotropy induced by the heat treatments in a magnetic field seems rather to decrease in magnitude with increasing titanium content. Furthermore, the electron microscopic observation has revealed that the precipitate particles developed by the optimal treatment are likely to increase in both size and dimension ratio with increasing titanium content.
\end{abstract}

(Received February 9, 1970)

\section{Introduction}

Many attempts have been made to improve the properties of Alnico alloys by the addition of titanium, and it has been found that its addition particularly contribute to the increase in coercive force of the alloys. Among those works, it is most noteworthy that Luteijn and de $\mathrm{Vos}^{(1)}$ reported that remarkable magnet characteristics of ${ }_{B} H_{c}=1300 \mathrm{Oe}$ and $(B H)_{\max }=11.0 \mathrm{MG} \mathrm{Oe}$ could be attained with a columnar specimen of Ticonal X $(34 \%$ $\mathrm{Co}, 14 \% \mathrm{Ni}, 7 \% \mathrm{Al}, 5 \% \mathrm{Ti}, 4 \% \mathrm{Cu}$ and balance $\mathrm{Fe})$ by isothermal treatment in a magnetic field. Recently Planchard et al. (2) have achieved a much higher coercivity, $2000 \mathrm{Oe}$, with Alnico-type alloys containing greater amounts of $\mathrm{Co}$ and $\mathrm{Ti}$ than Ticonal X. Such a high value of coercivity seems to be comparable with the highest one that can be estimated from the theory of ferromagnetic single-domain particles. These results have suggested the possibility of the practical production of magnets with higher energy products than $10 \mathrm{MGOe}$, thus stimulating an increasing interest in Alnico 8-type alloys containing a large amount of titanium; Alnico 8 is the name of a typical commercial magnet alloy, which is nominally composed of $35 \% \mathrm{Co}, 14.5 \% \mathrm{Ni}, 7 \% \mathrm{Ti}, 5 \%$ $\mathrm{Cu}$ and the balance $\mathrm{Fe}$, comparable with Ticonal X.

Now, the following questions would arise proposed about Alnico 8-type alloys: How can such type alloys have coercivities considerably higher than Alnico 5, and why can the high coercivity be achieved only through

* This paper was originally published in Japanese in J. Japan Inst. Metals, 33 (1969), 1314.

** Department of Applied Physics, Faculty of Engineering, Nagoya University, Nagoya, Japan.

***⿰冫⿰亅⿱丿丶丶 Nagoya University. Present address: Sumitomo Special Metals Co., L.td., Osaka, Japan.

(1) A. J. Luteijn and K. J.de Vos: Philips Res. Rep., 11 (1956), 489.

(2) E. Planchard, C. Bronner and J. Sauze : Proceeding of Journées Internationales des Applications du Cobalt, Brussels, (1964), 134.

Trans. J I M isothermal treatment in a magnetic field? In order to solve the Alnico 8 problem, it is necessary to clarify the transformations and the resulting structure in the Alnico 8-type alloys. However, little has been known about the metallurgical aspects of Alnico 8-type alloys ${ }^{(3) \sim(5)}$. In the present study, experiments were carried out to investigate the effects of titanium on the transformations and the microstructures of Alnico 8-type alloys of various compositions. As a first step to make clear the above questions, the magnet characteristics were measured, and metallurgical considerations were given to the results. Particularly the effect of titanium on the factors involved in the isothermal treatment in a magnetic field were examined in detail.

\section{Experimental Procedure}

Specimens of Alnico 8-type alloys were melted in vacuo with a high-frequency induction furnace and injection cast into a quartz tube 3 or $5 \mathrm{~mm}$ in diameter. The compositions of the specimens were varied in such a way as to contain $1 \sim 7.5 \%$ titanium and other elements in similar proportion to that of Alnico 8. The results of chemical analysis are shown in Table 1 . All the ingots were annealed in argon at about $1200^{\circ} \mathrm{C}$ to promote homogeneity. Then specimens were prepared into suitable shapes for the respective measurements.

Metallographic investigation was carried out mainly by micro-examination of specimens quenched from various temperatures. Particularly the details of the two-phase structures containing fine precipitate particles were examined by means of an electron microscope, where the oxide replica method was adopted ${ }^{(6)}$. Also measurements of resistivity at elevated temperatures were made at a

(3) A. J. J. Koch, M.G. vd Steeg and K. J.de Vos: Conf. Magnetism and Mag. Materials, (1956), 173.

(4) G. Vallier, C. Bronner and R. Peffen : Cobalt, 34 (1967), 10.

(5) C. Bronner, J. Sauze and E. Planchard : Cobalt, 36 (1967), 123.

(6) K.J.de Vos: Philips Res. Rep., 18 (1963), 405. 
rate of about $10^{\circ} \mathrm{C} / \mathrm{min}$ on specimens of $3 \mathrm{~mm}$ diameter and $80 \mathrm{~mm}$ length, to determine the critical temperature of the two-phase separation transformation; it can be most accurately determined according to a knick on the resistivity-vs.-temperature curve obtained. Thermomagnetic analyses were made by means of a magnetic balance with very small specimens, which may allow the measurement to be performed at a very rapid rate. Besides, with a specimen of $5 \mathrm{~mm}$ diameter and $16 \mathrm{~mm}$ length set into a bottle type of magnet, a $I-H$ loop was measured within the limit of an external field of 5000 Oe by a Cioffi-type recording fluxmeter. From this loop, a saturation induction $4 \pi I_{s}$ (by extrapolating $I-v s .-1 / H^{2}$ ), a residual induction $B_{r}$, a coercive force $I H_{c}$ and a maximum energy product $(B H)_{\max }$ were obtained.

Table 1 Chemical compositions of specimens

\begin{tabular}{|c|c|c|c|c|c|c|}
\hline \multirow{2}{*}{$\begin{array}{l}\text { Specimen } \\
\text { No. }\end{array}$} & \multicolumn{6}{|c|}{ Composition (wt\%) } \\
\hline & $\mathrm{Ti}$ & Al & $\mathrm{Ni}$ & Co & $\mathrm{Gu}$ & $\mathrm{Fe}$ \\
\hline 1 & 1.05 & 7.75 & 15.02 & 36.54 & 2.55 & Res. \\
\hline 2 & 2.10 & 7.30 & 14.69 & 35.81 & 2.62 & "I \\
\hline 3 & 3.10 & 7.45 & 14.91 & 35.44 & 2.49 & $" 1$ \\
\hline 4 & 3.64 & 7.08 & 14.47 & 35.47 & 2.70 & 11 \\
\hline 5 & 4.36 & 7.02 & 14.31 & 35.06 & 2.82 & " \\
\hline 6 & 5.48 & 6.95 & 14.36 & 34.33 & 2.54 & " \\
\hline 7 & 6.39 & 6.92 & 13.82 & 3.362 & 2.83 & "I \\
\hline 8 & 7.52 & 6.92 & 13.85 & 33.56 & 2.48 & $" 1$ \\
\hline
\end{tabular}

\section{Behaviours of Transformations}

Fig. 1 shows a constitutional diagram in the solid state for a series of the alloys investigated in this work, which were determined from the various measurements above described; the $\mathrm{Ti}$ content in the specimens is shown on the abscissa. The transformations occurring in the solid state of the Alnico 8-type alloys are very similar to those of Alnico 5 , that is $\alpha \rightleftarrows \alpha+\gamma$ ( $\mathrm{A}_{4}$ and $\mathrm{A}_{3}$ transformations) and $\alpha_{\leftarrow} \alpha_{1}+\alpha_{2}$ (two-phase separation transformation or miscibility gap reaction). As a result, the diagram consists of four regions, $\alpha, \alpha+\gamma ; \alpha_{1}+\alpha_{2}+\gamma$ and $\alpha_{1}+\alpha_{2}$, as shown in Fig. 1. The phase boundaries separating these regions indicate how the critical temperatures of the transformations change with the $\mathrm{T} i$ content. Besides, Curie points are shown corresponding to a homogeneous

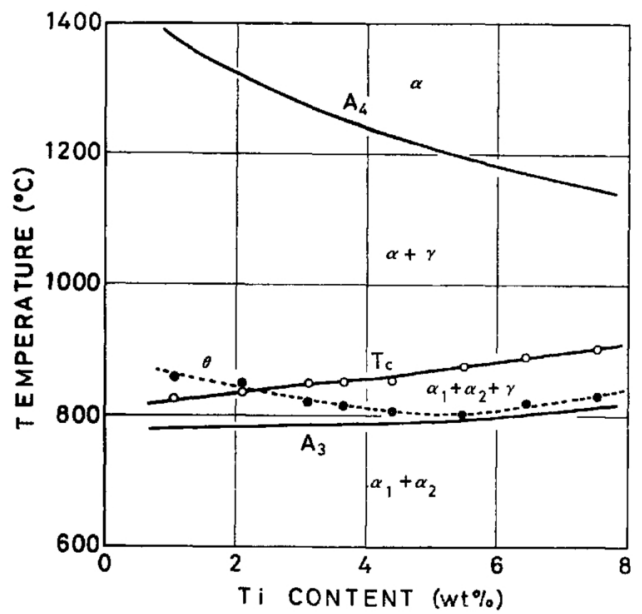

Fig. 1 Constitutional diagram in the solid state for Alnico 8-type alloys with various $\mathrm{Ti}$ contents. $\alpha$ phase state of specimens, which have been determined by thermomagnetic analyses.

\section{1. $\mathbf{A}_{4}$ and $\mathbf{A}_{3}$ transformations}

All the alloys consist of a homogeneous $\alpha(b c c)$ phase at higher temperatures. At temperatures below about $1300^{\circ} \sim 1200^{\circ} \mathrm{C}$, however, they precipitate the $\gamma(f c c)$ phase to transform into a two-phase state of $\alpha+\gamma$ which is called the $\mathrm{A}_{4}$ transformation. Then, below about 800 ${ }^{\circ} \mathrm{C}$ the $\gamma$ phase transforms again into the $\alpha$ phase, the $A_{3}$ transformation. The $A_{3}$ transformation cannot be prevented even by an extremely drastic quenching, but takes place in a martensitic form of transformation. Moreover, the $\gamma$ phase may precipitate also when the specimen is quenched from a homogeneous $\alpha$ region at higher temperatures, followed by annealing above the $A_{3}$ temperature.

The $\mathrm{A}_{4}$ temperature has been determined by microexamination of the quenched specimen during cooling from the $\alpha$ phase region. It is seen in Fig. 1 that the $A_{4}$ temperature considerably falls with increase in $\mathrm{Ti}$ content. On the other hand, the $A_{3}$ temperature has been determined by micro-examination of the specimen quenched on the way of heating, after a solution treatment in the $\alpha$ phase region followed by quenching to room temperature. The heating time was $48 \mathrm{hr}$ at each temperature. The $A_{3}$ temperature rises slightly with the $\mathrm{Ti}$ content. From the above results, it can be concluded that the phase region where the $\gamma$ phase is existing becomes narrower as the $\mathrm{Ti}$ content increases. This seems to be reasonable in accordance with the well-known fact that the titanium element is an $\alpha$ former in the ferrous alloys.

\section{Two-phase separation transformation}

The critical temperature of this transformation has been determined from the measurement of resistivity at elevated temperatures. As shown in Fig. 1, the critical temperature (hereafter, it is denoted as $T_{C}$ ) rises with the $\mathrm{Ti}$ content, reaching about $900^{\circ} \mathrm{C}$ at $7.5 \% \mathrm{Ti}$. Since the $T_{C}$ point lies above the $A_{3}$ temperature of the alloys, a three-phase region of $\alpha_{1}+\alpha_{2}+\gamma$ reveals itself between the two kinds of critical temperatures. However, the $\alpha \rightarrow \gamma$ transformation is much slower in the rate of transformation than the two-phase separation transformation. Accordingly, even if a specimen consisting of a homogeneous $\alpha$ phase is annealed just below the $T_{C}$ temperature for about $10 \mathrm{~min}$, the $\gamma$ phase is hardly observable and only the two-phase separation reaction will proceed.

\section{Magnetic transition}

In Fig. 2, thermomagnetic curves are shown for No. 5 specimen $(4.36 \% \mathrm{Ti})$ annealed for $48 \mathrm{hr}$ at $700^{\circ}, 650^{\circ}$ and $600^{\circ} \mathrm{C}$, respectively. Since the alloy consists of two phases of $\alpha_{1}$ and $\alpha_{2}$ after the annealing, each curve shows two inflexion points, which correspond to Curie points of the two phases. The lower one indicates a Curie point of the $\alpha_{2}$ phase, $\theta_{2}$, which falls as the annealing temperature is lowered. A similar behaviour has been observed in the case of Alnico $5^{(7)}$. This appears to imply that as the annealing temperature decreases, the equilibrium

(7) Y. Iwama : Trans. JIM, 8 (1967), 18. 
composition of the $\alpha_{2}$ phase involved shifts gradually toward higher contents of $\mathrm{Ni}, \mathrm{Al}$ and possibly $\mathrm{Ti}$. In Fig. 3 , the $\theta_{2}$ temperatures of all the alloys determined in the same manner as mentioned above are summarized against the Ti content. The descending trend of the $\theta_{2}$ can be observed with lowering annealing temperature for all the

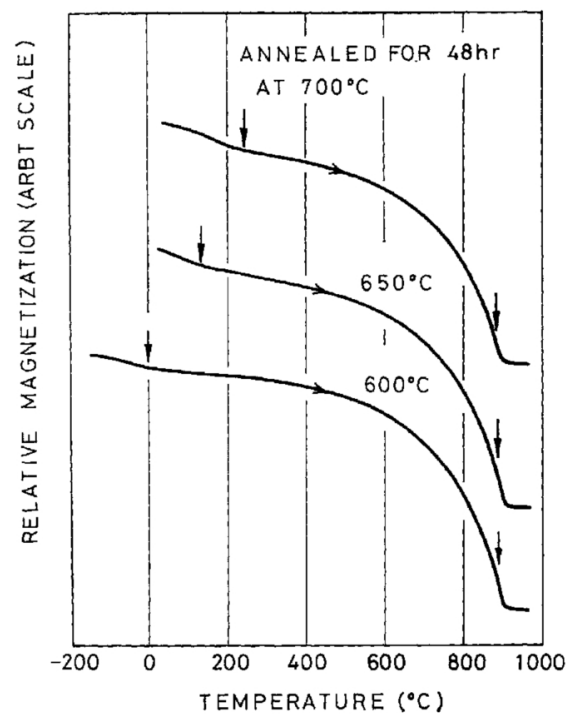

Fig. 2 Thermomagnetic curves of specimen No. $5(4.36 \% \mathrm{Ti})$ annealed for $48 \mathrm{hr}$ at various temperatures.

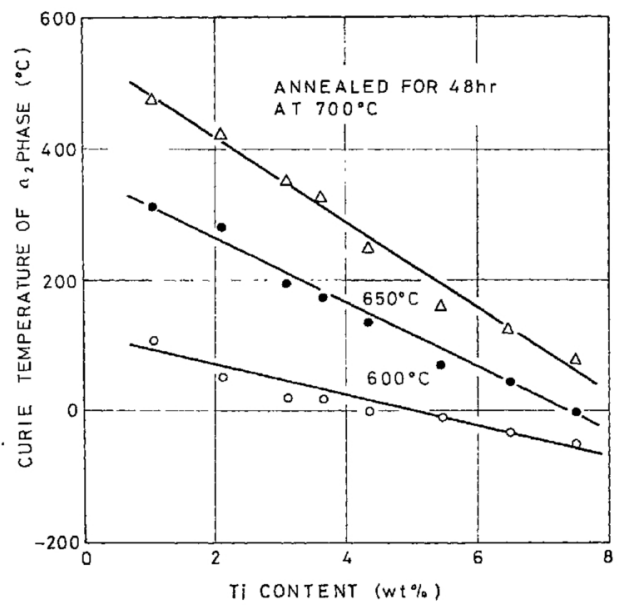

Fig. 3 Variation in Curie point of $\alpha_{2}$ phase with Ti content of specimens annealed at various temperatures.

alloys. It should deserve particular attention that $\theta_{2}$ decreases linearly with the $\mathrm{Ti}$ content if the specimens are annealed at a certain temperature. This fact seems to suggest that titanium may preferentially dissolve into the $\alpha_{2}$ phase, resulting in the decrease of its Curie point. Therefore, when the alloy containing more than $5 \% \mathrm{Ti}$ is annealed at $600^{\circ} \mathrm{C}$, the $\theta_{2}$ falls below room temperature ; that is, the $\alpha_{2}$ phase is non-magnetic at room temperature. This is a favourable condition for the Alnicotype magnet alloys ${ }^{(7)}$, because the $\alpha_{2}$ phase forms a matrix comprising ferromagnetic single-domain particles of the $\alpha_{1}$ phase, as will be shown later. It does illustrate the meaning of the aging treatment at $600^{\circ} \mathrm{C}$ which is also very important in the Alnico 8-type alloys.

The other Curie point, which is observed in the higher temperature side in Fig. 2, lies at about $890^{\circ} \mathrm{C}$ independent of the annealing temperature. Though it appears to show a Curie point of the $\alpha_{1}$ phase, it is not the case. It is because the upper Curie point is situated above its own $T_{C}$ temperature for almost all the alloys, so that during the measurement with an ordinary heating rate the dissolving reaction, $\alpha_{1}+\alpha_{2} \rightarrow \alpha$, may probably proceed in some extent before the $\theta_{1}$ is reached. In order to obtain the accurate $\theta_{1}$ of the equilibrium $\alpha_{1}$ phase, therefore, it is necessary to make a thermomagnetic measurement at a heating rate as rapid as possible, after the specimen is pre-annealed at a specific temperature. Even in the case of Fig. 2 in which thermomagnetic analyses were performed at a fairly rapid rate (over 100 ${ }^{\circ} \mathrm{C} / \mathrm{min}$ ), it seemed to be still insufficient.

In a similar way, the Curie point of the $\alpha$ phase, $\theta$, must be determined from the thermomagnetic curve which is measured by rapid cooling from a homogeneous $\alpha$ region. As previously noted, the $\theta$ 's shown in Fig. 1 have been determined by thermomagnetic analyses with a fairly rapid cooling rate. Nevertheless, since the $T_{C}$ lies above the $\theta$ except for the alloy with less than $2 \% \mathrm{Ti}$ content, the two-phase separation reaction probably takes place during cooling from the high temperatures before the $\theta$ is reached. In such cases, the apparent $\theta$ might be somewhat higher than the true $\theta$ otherwise obtained.

\section{Heat-Treatments and Magnetic Characteristics}

It has been generally recognized that in the case of Alnico 8 or Ticonal $\mathrm{X}$, the isothermal treatment in a magnetic field (hereafter, it will be abbreviated as IFT) at about $800^{\circ} \mathrm{C}$ is much more effective than the field cooling treatment, which has been exclusively applied to Alnico $5^{(1)(8)}$. Particularly it can bring about much higher coercivity. Therefore, the IFT has been applied to a series of alloys with different $\mathrm{Ti}$ contents, so as to investigate the influences of both the temperature and the time of the IFT upon the resulting magnet characteristics.

After the solution treatment in a homogeneous $\alpha$ region, the specimens recieved the IFT at various temperatures of $880^{\circ} \sim 750^{\circ} \mathrm{C}$ for 3,5 or $10 \mathrm{~min}$, respectively. Subsequently, after the aging treatment at 600 ${ }^{\circ} \mathrm{C}$ for $16 \mathrm{hr}$, their magnet characteristics were measured. In Fig. 4, as an example, the coercive force is shown against the holding time of the IFT for No. 7 specimen $(6.38 \% \mathrm{Ti})$. It is easily seen that the IFT at $840^{\circ} \mathrm{C}$ for $5 \mathrm{~min}$ leads to the highest coercive force of more than $1800 \mathrm{Oe}$. When the IFT is carried out at a temperature higher than $850^{\circ} \mathrm{C}$, the coercive force obtained decreases monotonously with the treatment time. And the higher the temperature of the IFT, the lower becomes the coercive force. On the other hand, when the IFT is carried out at a temperature lower than $840^{\circ} \mathrm{C}$, the situation is reversed. As the temperature of the IFT is lowered, the coercive force decreases, while the coercivity decreases monotonously with the treatment time in the same manner as in the former case. Thus, the coercive

(8) A.J. J. Koch, M. G. vd Steeg and K. J. de Vos: Berichte der Arbeitsgemeinschaft Ferromagnetismus, (1959), 130. 
force obtained with the IFT depends upon the temperature as well as the time of the treatment, and it should be emphasized that the temperature produces a much more significant influence. A similar tendency can be observed for other specimens. With respect to the time, on the contrary, about 5 to 10 min might probably be selected for all the specimens without any serious miscarrige.

Then, the specimens with different Ti contents were subjected to the isothermal treatment for a fixed time of $5 \mathrm{~min}$ at various temperatures with or without a magnetic field. The coercive forces obtained after the aging at $600^{\circ} \mathrm{C}$ for $16 \mathrm{hr}$ are shown in Fig. 5, where each curve shows a relation between the IFT temperature and the coercive force for the respective specimen. In every case,

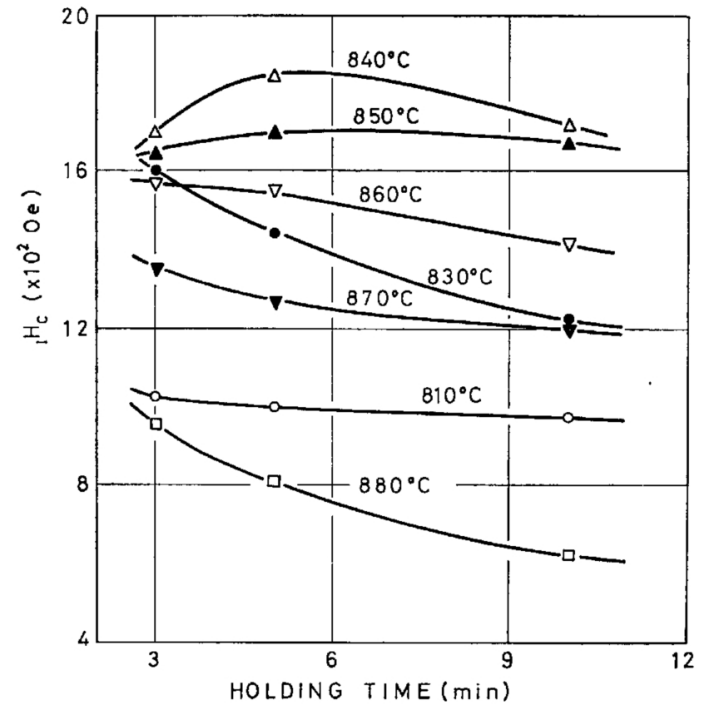

Fig. 4 Variation in coercive force with the holding time of the isothermal treatment at various temperatures for specimen No. $7(6.39 \% \mathrm{Ti})$.

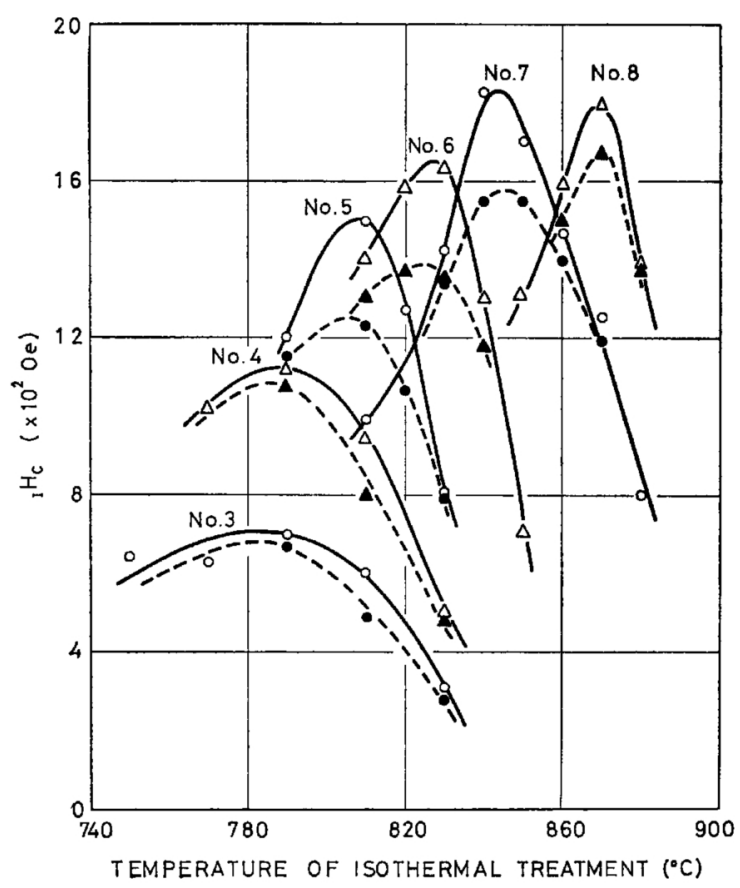

Fig. 5 Effect of temperature of the isothermal treatment for $5 \mathrm{~min}$ with or without a magnetic field on coercive force of $\mathrm{Ti}$-containing Alnico alloys, measured after aging for $16 \mathrm{hr}$ at $600^{\circ} \mathrm{C}$. the coercive force is obviously shown to reach a maximum at a certain temperature of the IFT, which is to be noted as the optimum isothermal treatment temperature, $T_{0}$. It can easily be seen that the $T_{0}$ shifts toward the higher temperature side as the $\mathrm{Ti}$ content increases. Moreover, the curves display the sharper and higher maxima with increasing $\mathrm{Ti}$ content, which, suggests that when the IFT is applied to the Alnico 8-type alloys high in Ti content, it is very significant to decide precisely the optimum temperature at which the treatment should be performed.

In Fig. 6, the magnet characteristics of $B_{r},{ }_{B} H_{C}$ and $(B H)_{\max }$ are shown against the $\mathrm{Ti}$ content for a series of alloys subjected to the optimum IFT. As the Ti content increases, ${ }_{B} H_{C}$ remarkably increases to a level of over $17000 \mathrm{e}$ at about $6 \% \mathrm{Ti}$, and then slightly decreases. On the contrary, $B_{r}$ decreases linearly with the Ti content. As a result, $(B H)_{\max }$ reaches a maximum at about $5 \% \mathrm{Ti}$, which corresponds to the composition of Alnico 8. In other words, as long as the composition of the Alnico 8type alloys is varied only in the $\mathrm{Ti}$ content, the alloy with about $5 \% \mathrm{Ti}$ may achieve the highest value of $(B H)_{\max }$, which amounts to about 5.5 MG Oe with the equiaxed cast structure.

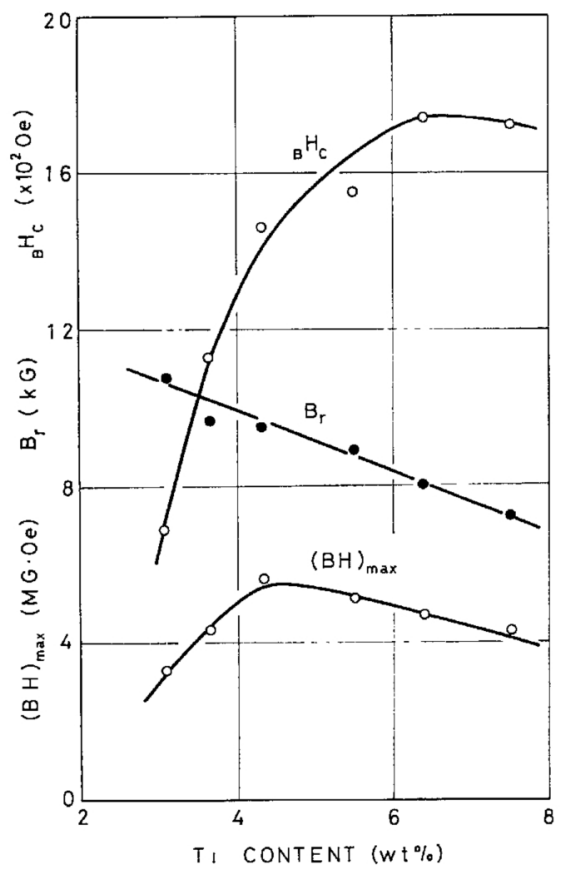

Fig. 6 Relation between magnetic properties and $\mathrm{Ti}$ content in Alnico 8-type alloys under the optimal isothermal treatment.

Fig. 5 also allows a comparison between the IFT and the isothermal treatment without a magnetic field. With respect to the optimum temperature i. e. the peak temperature of the curve, a similar tendency can be recognized for all the specimens. However, the extent of the increase in coercive force due to the field application changes in accordance with the $\mathrm{Ti}$ content in the specimens. And the most striking effect of a magnetic field can be observed in the alloy with about $5 \% \mathrm{Ti}$ or Alnico 8. In Fig. 7, $4 \pi I_{S}$ and $B_{r} / 4 \pi I_{S}$ are plotted against the Ti content in either case of the optimum IFT or the optimum isothermal treatment without a magnetic 


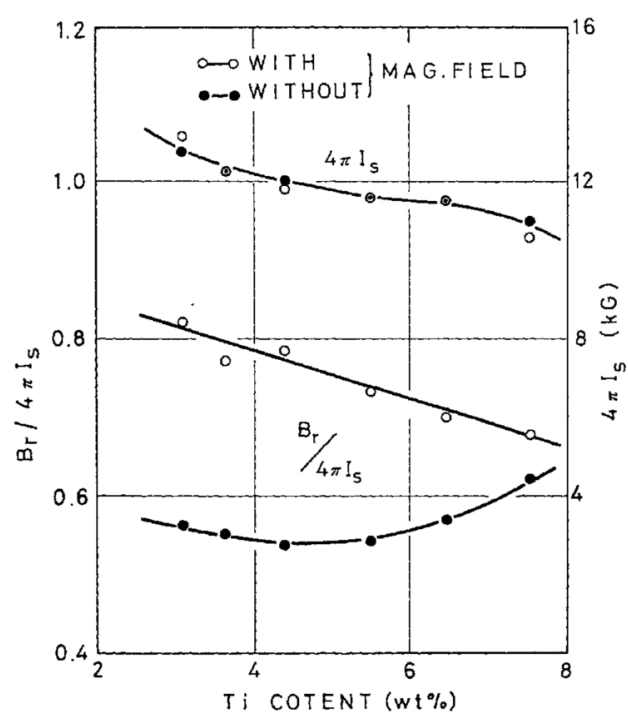

Fig. 7 Relation between Ti content and saturation induction as well as loop squareness factor in Alnico 8-type alloys under the optimal isothermal treatment with or without magnetic field.

field. These results would make it possible to more closely examine the efficiency of a magnetic field during the treatment. Since it is evident that $4 \pi I_{S}$ is independent of the application of a magnetic field within the experimental error for all the specimens, the ratio of $B_{r} / 4 \pi I_{S}$ may be regarded as a certain measure for the efficiency of a magnetic field or the magnitude of the magnetic anisotropy induced by the field treatment.

Upon application of a magnetic field, this ratio exceeds 0.8 with the lower $\mathrm{Ti}$ contents, but it obviously decreases with increasing Ti content. This fact appears to imply that the addition of titanium rather deteriorates the induced magnetic anisotropy or the squareness factor of the demagnetizing curve. On the other hand, in the case without a magnetic field, the ratio is a little higher than 0.5 , which might be theoretically estimated. It is to be noticed, however, that it increases above 0.6 for the alloys with higher Ti contents. This fact seems to be attributed partly to some texture which has been produced in the specimens during casting, though the ample reasons have been still unclarified.

\section{Discussions}

The process through which the transformations take place in Alnico 8-type alloys is quite similar to that in Alnico $5^{(9)}$. A mechanism of magnetic hardening, therefore, can be explained in an analogous manner for both types of Alnico alloys by applying the theory of spinodal decomposition ${ }^{(10)}$ to the two-phase separation reaction involved in them. It is to be noted, however, that in the case of Alnico 8-type alloys the spinodal decomposition of the $\alpha$ phase can be made most favourably through the isothermal annealing for a short time (about $10 \mathrm{~min}$ ) at an optimum temperature. From the theoretical standpoint of spinodal decomposition, this mode of treatment may probably be more reasonable than

(9) C. Bronner, J-P. Haberer, E. Planchard and J. Sauze : Cobalt, $40(1968), 131$.

(10) J. W. Cahn : Act. Met., 9 (1961), 795. the controlled cooling treatment which is generally used for Alnico 5-type alloys. It can also be expected that the average wavelength of the concentration fluctuation in the $\alpha$ phase, i. e. the diameter of precipitate particles, will be reduced as the annealing temperature is lowered. Moreover, if the treatment is performed in a magnetic field, the elongated fine particles of a strongly ferromagnetic $\alpha_{1}$ phase may precipitate in the matrix of the non-magnetic $\alpha_{2}$ phase in a regular array parallel to the field direction.

Now, it is considered that immediately after the IFT for a duration of several $\mathrm{min}$, the precipitate particles have already reached almost a final height of perfection in both size and shape. In this stage, however, the matrix phase is not yet so sufficiently rich in $\mathrm{Ni}, \mathrm{Al}$ and $\mathrm{Ti}$ as to be non-magnetic at room temperature, as described in III-3. Accordingly, its coercive force must be comparatively low at room temperature ${ }^{(7)}$. But, if the aging treatment is carried out at $600^{\circ} \mathrm{C}$ for $10 \sim 20 \mathrm{hr}$ after the IFT, both constituents of $\alpha_{1}$ and $\alpha_{2}$ can achieve their final equilibrium compositions and consequently a favourable situation may be accomplished in which the $\alpha_{1}$ phase is strongly ferromagnetic and the $\alpha_{2}$ phase is non-magnetic at room temperature (cf. Fig. 3 ). It has been proved that its coercive force can be largely improved during the aging.

Since the aging is performed at a rather low temperature in the vicinity of $600^{\circ} \mathrm{C}$, the atomic diffusion involved in the process may take place within a relatively short range. which is assumed to take an order of the diameter of the precipitate particle. It follows that any appreciable change will not occur in the precipitate structure. This has been confirmed by electron microscopy of the structures. Photos. I (a) $\sim(d)$ are the results obtained on (100) and (001) planes of a single crystal of Alnico 8 , showing the precipitate structures before and after the aging, respectively. According to de $\mathrm{Vos}^{(6)}$, the bright area in the photographes represents the $\alpha_{1}$ phase, and the dark area, the $\alpha_{2}$ phase which is rich in $\mathrm{Ni}, \mathrm{Al}$ and Ti. It is noteworthy that by the aging, the coercive force of this specimen has increased twice that before aging. Besides, a closer inspection of the photographs reveals the presence of very fine secondary precipitates in the matrix after the aging (cf. Photos. $1(\mathrm{~b})$ and (d)), which seem to be formed during the aging.

Now, the influence of titanium upon the isothermal field treatment will be discussed. In Fig. 8, the optimum IFT temperature $\left(T_{0}\right)$, the critical temperature of the two-phase separation $\left(T_{C}\right)$ and Curie points of the initial $\alpha$ and the final $\alpha_{1}$ phase $\left(\theta\right.$ and $\left.\theta_{1}\right)$ are plotted against the $\mathrm{Ti}$ content in the specimens. The $T_{0}$ lies about $50^{\circ} \mathrm{C}$ below the $T_{C}$, independent of the Ti content. This suggests that the most significant factor to decide the $T_{0}$ temperature is the $T_{c}$ of the alloy. Furthermore, an electron microscope study of various specimens has shown that the diameter of the precipitate particle does not depend on the $\mathrm{Ti}$ content but merely on the IFT temperature. It follows that the two-phase structure which is brought about by the optimum IFT grows coarser with increase in $\mathrm{Ti}$ content, because with increasing $\mathrm{Ti}$ content, the higher optimum IFT temperature is selected. Photos. 


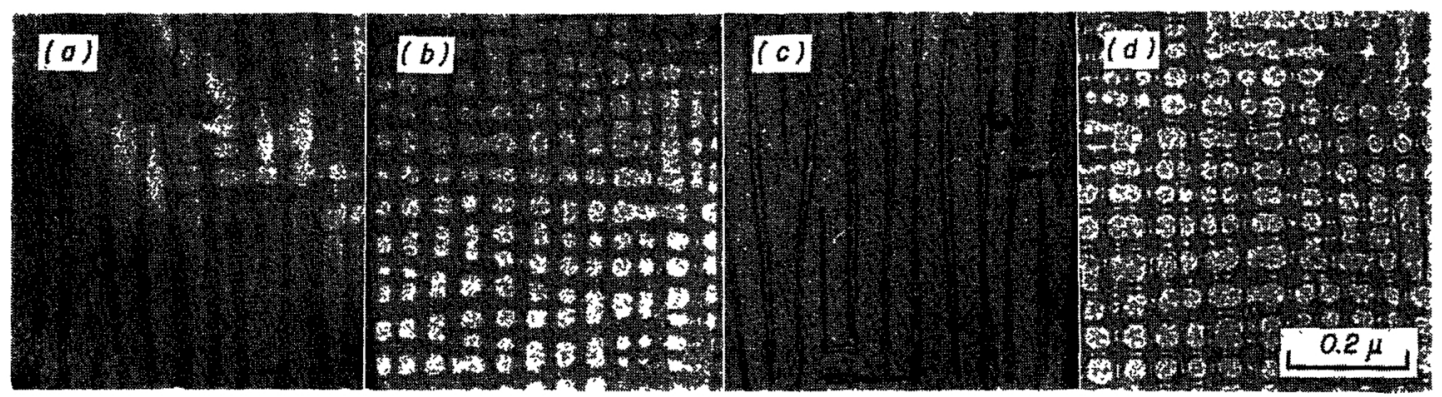

Photo. 1 Electron micrographs of single crystalline Alnico 8, isothermally treated for 30 min at $800^{\circ} \mathrm{C}$ with magnetic field along the $[001]$ axis, followed by aging for $16 \mathrm{hr}$ at $600^{\circ} \mathrm{C}$; (a) and (b) are before aging, and (c) and (d) after aging. $\begin{array}{llll}\text { (a) }(100) & \text { (b) }(001) & \text { (c) }(100) & \text { (d) }(001)\end{array}$

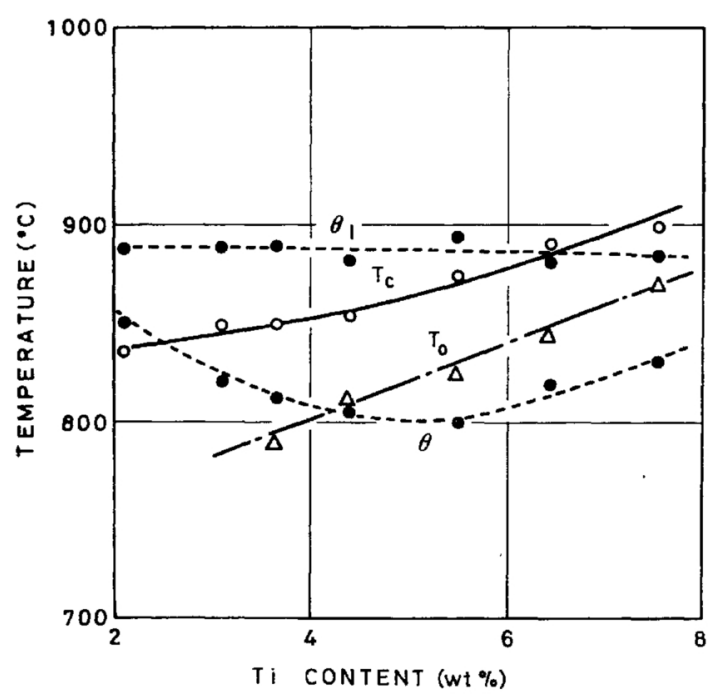

Fig. 8 Variations in critical temperature of miscibility gap reaction, optimal temperature of the isothermal treatment and Curie points of $\alpha$ and $\alpha_{1}$ phases with Ti content in Alnico 8-type alloys. larger in dimension ratio than those in Alnico 5 . However, since these observations have been carried out by means of the oxide replica method for polycrystalline specimens, the above results on their diameter and dimension ratio should be regarded as rather semiquantitative ones.

Conclusively, it can be seen from Fig. 8 that for the specimens with higher Ti contents, $\theta<T_{0}<\theta_{1}$. Therefore, when a specimen is quenched from a homogeneous $\alpha$ region down to the $T_{0}$ temperature where the two-phase separation may set in, it will be paramagnetic in the earliest stage. Consequently, it is likely that the decomposition would take place rather in a isotropic mode at least in the earlier stage, though its duration might be so short. As the decomposition advances in some extent, the precipitates of the $\alpha_{1}$ phase is enriched in Fe and $\mathrm{Co}_{0}$ enough to be ferromagnetic even at the $T_{0}$ temperature. Then, in virtue of an interaction between the ferromagnetic moments and the applied field, the structure will be altered to an anisotropic structure with particles

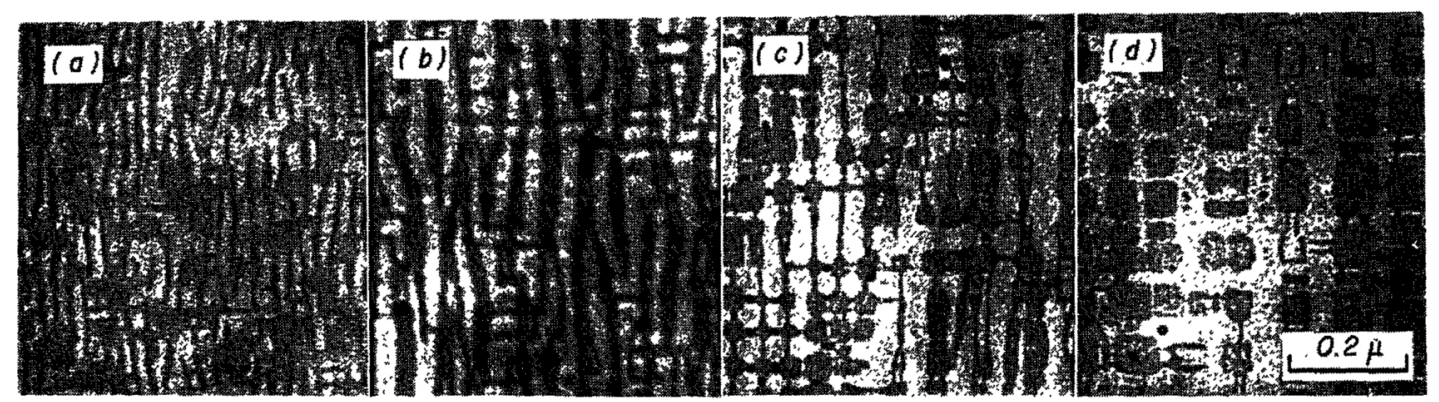

Photo. 2 Electron micrographs of Alnico 8-type alloys containing various Ti content, after the optimal isothermal treatment followed by aging.

$\begin{array}{lllll}\text { (a) No. } 3(3.10 \% \mathrm{Ti}) & \text { (b) No. } 5(4.36 \% \mathrm{Ti}) & \text { (c) No.7 }(6.39 \% \mathrm{Ti}) & \text { (d) No. } 8(7.52 \% \mathrm{Ti})\end{array}$

$2(\mathrm{a}) \sim(\mathrm{d})$ show the structures obtained by the optimum treatment for some of the specimens with various $\mathrm{Ti}$ contents. Since the field cooling treatment of the specimen with $3.10 \% \mathrm{Ti}$ gives a coercive force higher than by the IFT, the structure brought about by the field cooling treatment with a rate of about $1^{\circ} \mathrm{C} / \mathrm{sec}$ is shown in the photograph. From these photographs it is clear that as the $\mathrm{Ti}$ content increases from 3.10 to $7.52 \%$, the particles grow larger from 200 to $500 \AA$ in average diameter. Thus it is shown that the precipitate particles in the high titanium Alnico are much larger in average diameter than those in Alnico 5 (about $150 \AA$ ). It is also noted that the particles in the high titanium Alnico are by far elongated in the field direction ${ }^{(11)}$. Since in the high titanium Alnico $T_{C}$ and $T_{0}$ are much higher than $\theta$, it seems likely that the earlier isotropic decomposition is sufficiently advanced and consequently disturbs the subsequent anisotropic alteration effect due to the applied field.

Recently high titanium Alnico magnet alloys have become the object of increasing attention on a global level. The present work is concerned with a series of Alnico 8type alloys in which only the $\mathrm{Ti}$ content is varied. Further attempts will be made to solve the high titanium Alnico problems in order to contribute to the development of a higher quality of magnet alloys.

(11) J. W. Cahn : J. Appl. Phys., 34(1963), 3581. 


\section{Summary}

In the present work, a series of Alnico 8-type alloys have been investigated in which only the titanium content was changed and the other chemical compositions were kept in the same proportion as in the commercial magnet alloy, Alnico 8. Micro-examination of the structures, thermomagnetic analyses and measurements of resistivity at elevated temperatures and magnet characteristics were carried out to investigate the relationships between the transformations such as the magnetic transition and the titanium content and also to clarify the mechanism of magnetic hardening in the Alnico 8-type alloys. Furthermore, factors involved in the isothermal field treatment were investigated for these alloys, and particularly influences of the temperature and the time of the treatment upon magnetic characteristics were closely examined with various titanium contents. The results obtained in the present work are summarized in the following:

(1) On the whole, behaviours of transformations and the mechanism of magnetic hardening in Alnico 8-type alloys are very similar to those in Alnico 5, as have been so far clarified.

(2) With increasing titanium content, the $\alpha \rightarrow \gamma$ transition temperature gradually falls so that the precipitation of the $\gamma$ phase is limited to a narrow temperature range.

(3) The critical temperature for the two-phase separation transformation rises with the titanium content.

(4) For these alloys with more than $2 \% \mathrm{Ti}$, the Curie point of the homogeneous $\alpha$ phase lies below the critical temperature of the two-phase separation transformation. And the Curie point of the $\alpha_{1}$ phase is probably situated above $890^{\circ} \mathrm{C}$ independent of the titanium content. On the other hand, the Curie point of the $\alpha_{2}$ phase markedly falls as the annealing temperature is lowered. Also, with increasing titanium content, the Curie point of the $\alpha_{2}$ phase falls. This seems to imply that the titanium content is dissolved preferentially into $\alpha_{2}$ phase in the same manner as aluminum and nickel.

(5) The optimum time of isothermal magnetic treatment is $5 \sim 10 \mathrm{~min}$, but the coercive force achieved does not depend largely upon the time of the treatment. On the contrary, the coercivity depends profoundly upon the temperature of the treatment, and especially the alloys with over $5 \% \mathrm{Ti}$ are so sensitive that the treatment should be performed at the optimum temperature within an error of $\pm 10^{\circ} \mathrm{C}$.

(6) The optimum temperature of the treament appears to lie about $50^{\circ} \mathrm{C}$ below its own critical temperature of the two-phase separation transformation, and it rises gradually with increasing titanium content.

(7) Among the magnetic characteristics achieved with the optimum treatment, the coercive force remarkably increases as the titanium content increases up to about $5 \%$, while the saturation induction and the remanence decrease linearly with the titanium content. It follows that a maximum energy prcduct reaches a maximum at about $5 \% \mathrm{Ti}$, which corresponds to the titanium content in Alnico 8.

(8) From an electron microscopic examination on the precipitate structures obtained with the optimum treatment, it has been found that the precipitate particles become larger in diameter and in dimension ratio with increasing titanium content.

(9) Magnetic anisotropy induced by the applied field during the treatment appears to be rather reduced with increasing titanium content.

\section{Acknowledgment}

The authors gratefully acknowledge that this work was partly supported by the grant-in-aid for scientific research of the Education Ministry. 